\title{
ON THE LONG TIME BEHAVIOR OF SOLUTIONS TO THE INTERMEDIATE LONG WAVE EQUATION
}

\author{
CLAUDIO MUÑOZ, GUSTAVO PONCE, AND JEAN-CLAUDE SAUT
}

\begin{abstract}
We show that the limit infimum, as time $t$ goes to infinity, of any uniformly bounded in time $H^{3 / 2+} \cap L^{1}$ solution to the Intermediate Long Wave equation converge to zero locally in an increasing-in-time region of space of order $t / \log (t)$. Also, for solutions with a mild $L^{1}$-norm growth in time is established that its limit infimum converge to zero, as time goes to infinity. This confirms the non existence of breathers and other solutions for the ILW model moving with a speed "slower" than a soliton. We also prove that in the far field linearly dominated region, the $L^{2}$ norm of the solution also converges to zero as time approaches infinity.

In addition, we deduced several scenarios for which the initial value problem associated to the generalized Benjamin-Ono and the generalized Intermediate Long Wave equations cannot possess time periodic solutions (breathers).

Finally, as it was previously demonstrated in solutions of the KdV and BO equations, we establish the following propagation of regularity result : if the datum $u_{0} \in H^{3 / 2+}(\mathbb{R}) \cap$ $H^{m}\left(\left(x_{0}, \infty\right)\right)$, for some $x_{0} \in \mathbb{R}, m \in Z^{+}, m \geq 2$, then the corresponding solution $u(\cdot, t)$ of the Intermediate Long Wave equation belongs to $H^{m}(\beta, \infty)$, for any $t>0$ and $\beta \in \mathbb{R}$.
\end{abstract}

\section{INTRODUCTION AND MAIN RESULtS}

We consider the Intermediate Long Wave (ILW) equation in 1D for $\delta>0$,

$$
\partial_{t} u+\mathcal{T}_{\delta} \partial_{x}^{2} u+\frac{1}{\delta} \partial_{x} u+u \partial_{x} u=0, \quad(t, x) \in \mathbb{R} \times \mathbb{R} .
$$

Here and along this paper $u=u(t, x) \in \mathbb{R}$ is a real-valued function, and

$$
\mathcal{T}_{\delta} f(x):=-\frac{1}{2 \delta} \text { p.v. } \int \operatorname{coth}\left(\frac{\pi(x-y)}{2 \delta}\right) f(y) d y .
$$

Note that $\mathcal{T}_{\delta}$ is a order zero Fourier multiplier, in the sense that $\partial_{x} \mathcal{T}_{\delta}$ is the multiplier with symbol

$$
\sigma\left(\partial_{x} \mathcal{T}_{\delta}\right)=\widehat{\partial_{x} \mathcal{T}_{\delta}}=-2 \pi \xi \operatorname{coth}(2 \pi \delta \xi)
$$

The equation ILW (1.1) describes the long internal gravity waves in a two layers stratified fluid, the lower layer having a large finite depth represented by the parameter $\delta$, see [10],

Date: October 10, 2019.

2000 Mathematics Subject Classification. Primary 37K15, 35Q53; Secondary 35Q51, 37K10.

Key words and phrases. Intermediate long wave equation, decay estimates, virial.

CM was partially supported by Fondecyt no. 1191412, Ecos-Sud C18E06 and Conicyt PIA AFB170001. J.-C. S. is partially supported by the ANR Project ANuI. 
[21], [11], [6], [30] for a formal derivation and [5, 7] for a rigorous derivation in the sense of consistency.

In [1] it was proven that solutions of the ILW as $\delta \rightarrow \infty$ (infinite depth limit) converge to solutions of the Benjamin-Ono (BO) equation, see [4], [28]

$$
\partial_{t} u+\mathcal{H} \partial_{x}^{2} u+u \partial_{x} u=0
$$

with the same initial data, here $\mathcal{H}$ stands for the Hilbert transform

$$
\begin{aligned}
\mathcal{H} f(x) & :=\frac{1}{\pi} \mathrm{p} \cdot \mathrm{v} \cdot\left(\frac{1}{x} * f\right)(x) \\
& :=\frac{1}{\pi} \lim _{\epsilon \downarrow 0} \int_{|y| \geq \epsilon} \frac{f(x-y)}{y} d y=(-i \operatorname{sgn}(\xi) \widehat{f}(\xi))^{\vee}(x) .
\end{aligned}
$$

Also, in [1] it was shown that if $u_{\delta}(t, x)$ denotes the solution of the ILW equation (1.1), then

$$
v_{\delta}(t, x)=\frac{3}{\delta} u_{\delta}\left(x, \frac{3}{\delta} t\right)
$$

converges as $\delta \rightarrow 0$ (shallow-water limit) to the solution of the Korteweg-de Vries (KdV) equation $[17]$

$$
\partial_{t} u+\partial_{x}^{3} u+u \partial_{x} u=0
$$

with the same initial data.

The ILW equation has been proven to be complete integrable. In fact the Inverse Scattering formalism was given in $[15,16]$, where one finds in particular the Lax pair of the ILW equation, but no rigorous theory for solving the Cauchy problem by this method is known (see however [14] for recent progress on the direct scattering problem). For further comments on general properties of the ILW equation we refer to [11], [15], [21], [29], and a recent survey [31].

Note also that the ILW equation is obtained as a one-dimensional, uni-directional reduction of a class of ILW systems derived in [5, 7]. Long time existence results for those systems were obtained in [32] but the global existence of even small solutions is still unknown to our knowledge.

Formally, real solutions of (1.1) satisfy infinitely many conservation laws, due to its integrability, (see eg [23], [24]) . The first three are the following ones, and are only due to the hamiltonian nature of the ILW equation.

$$
\begin{aligned}
I_{1}(u):= & \int u(t, x) d x=I_{1}\left(u_{0}\right), \quad I_{2}(u):=\int u^{2}(t, x) d x=I_{2}\left(u_{0}\right), \\
I_{3}(u):= & \int\left(u \mathcal{T}_{\delta} \partial_{x} u+\frac{1}{\delta} u^{2}-\frac{1}{3} u^{3}\right)(t, x) d x=I_{3}\left(u_{0}\right) \\
& \quad \text { (conservation of the Hamiltonian). }
\end{aligned}
$$

The first non-trivial invariant controls the $H^{1}$ norm of the solution and is given by 


$$
\begin{aligned}
I_{4}(u)=\int_{-\infty}^{\infty}( & \frac{1}{4} u^{4}+\frac{3}{2} u^{2} \mathcal{T}_{\delta}\left(u_{x}\right)+\frac{1}{2} u_{x}^{2}+\frac{3}{2}\left[\mathcal{T}_{\delta}\left(u_{x}\right)\right]^{2} \\
& \left.+\frac{1}{\delta}\left(\frac{3}{2} u^{3}+\frac{9}{2} u \mathcal{T}_{\delta}\left(u_{x}\right)\right)+\frac{3}{2 \delta^{2}}\right) d x
\end{aligned}
$$

The next invariant controls the $H^{3 / 2}$ norm of the solution. The general structure of the invariants is given for instance in [1].

The global well-posedness of the initial value problem (IVP) associated to the ILW equation (1.1) was studied in [1] where the following result was established:

Theorem A. [1] For any initial data $u_{0} \in H^{s}(\mathbb{R}), s>3 / 2$, for any $T>0$ and any $\delta>0$ the corresponding IVP associated to the ILP equation (1.1) has a unique solution

$$
u \in C\left([0, T]: H^{s}(\mathbb{R})\right) \cap C^{1}\left([0, T]: H^{s-2}(\mathbb{R})\right) \cap L^{\infty}\left([0, T]: H_{l o c}^{s+1 / 2}(\mathbb{R})\right) .
$$

Moreover, the map data $\rightarrow$ solution is locally continuous from $H^{s}(\mathbb{R})$ to the class defined in (1.10) and the $H^{n}(\mathbb{R}), n=0, \frac{1}{2}, 1, \frac{3}{2}$ norms of $u(\cdot, t)$ are uniformly bounded in time.

Remark 1.1. The proof uses the local $H^{s}(\mathbb{R}), s>3 / 2$ theory, the uniform $H^{3 / 2}(\mathbb{R})$ bound (thus integrability) and an interpolation argument.

Stronger results has been obtained in [25], where (unconditional) local well-posedness was established in $H^{s}(\mathbb{R})$ with $s \geq 1 / 2$. Due to the conservation of the hamiltonian, this result implies the global well-posedness of ILW in the energy space $H^{1 / 2}(\mathbb{R})$.

It is well-known that (1.1) possesses soliton (or solitary wave) solutions of the form [10]

$$
u(t, x)=Q_{\delta, c}(x-c t), \quad c>\frac{1}{\delta}
$$

where $Q_{\delta, c}$ solves

$$
\partial_{x} \mathcal{T}_{\delta} Q_{\delta, c}+\left(\frac{1}{\delta}-c\right) Q_{\delta, c}+\frac{1}{2} Q_{\delta, c}^{2}=0
$$

We remark that $Q_{\delta, c}$ is exponentially localized in space contrary to the BO soliton, and it is given by the formula [3]

$$
Q_{\delta, c}(s):=\frac{b(c) \sin (a(c) \delta)}{\cos (a(c) \delta)+\cosh (a(c) s)}, \quad a, b \text { depending on } c .
$$

The uniqueness (up to translation) of the ILW soliton is proven in $[2,3]$. Because of its integrability, the ILW equation possesses also multi-solitons, see [11].

Let $C>0$ be an arbitrary constant and $I_{b}(t)$ be the time-depending interval

$$
I_{b}(t):=\left(-\frac{C|t|^{b}}{\log |t|}, \frac{C|t|^{b}}{\log |t|}\right), \quad|t| \geq 10, \quad b \in(0,1] .
$$

Our first result here is the following : 
Theorem 1.1. Let $u=u(t, x)$ be a solution to (1.1) such that

$$
u \in C\left(\mathbb{R}: H^{s}(\mathbb{R})\right) \cap C^{1}\left([0, T]: H^{s-2}(\mathbb{R})\right) \cap L^{\infty}\left([0, T]: H_{l o c}^{s+1 / 2}(\mathbb{R})\right), \quad s>3 / 2 .
$$

Assume that

$$
u \in L_{l o c}^{\infty}\left(\mathbb{R}: L^{1}(\mathbb{R})\right)
$$

such that for some $a \in\left[0, \frac{1}{2}\right)$ and $c=c(\delta)>0$

$$
\sup _{t \in[0, T]} \int|u(t, x)| d x \leq c\langle T\rangle^{a}=c\left(1+T^{2}\right)^{a / 2} .
$$

Then for $b=1-a$,

$$
\liminf _{t \rightarrow \infty} \int_{|x| \leq \frac{C t^{b}}{\log (t)}}\left(u^{2}+\left(q_{\delta}\left(\partial_{x}\right) u\right)^{2}\right)(t, x) d x=0 .
$$

where $q_{\delta}\left(\partial_{x}\right)$ is a Fourier multiplier with even symbol $q_{\delta}(\xi), q_{\delta}(\xi)>0$ for $\xi \neq 0$ with $q_{\delta}(0)=0, q_{\delta}^{\prime}\left(0^{+}\right)=\delta^{-1 / 2}$, and

$$
q_{\delta}(\xi)=\frac{\xi}{\delta^{1 / 2}}+O\left(\frac{\xi^{3}}{\delta^{5 / 2}}\right) \quad \text { as } \xi \downarrow 0, \quad q_{\delta}(\xi)=\sqrt{\xi}+O\left(\frac{1}{\delta \xi^{1 / 2}}\right) \text { as } \xi \uparrow \infty .
$$

Therefore, no soliton nor breather solution exists for ILW inside the region $I(t)$, for any time $t$ sufficiently large.

Remark 1.2. The result in (1.18) also holds as $t \rightarrow-\infty$.

Remark 1.3. Related with the present problem we have the variants found in [18], [19], [22]. However, here as in [27] even under the strongest hypothesis $a=0$ in (1.17) one needs a weight outside the cut-off function $\phi(\cdot)$. This can be seen as a consequence of the weak dispersive relation in the ILW equation which does not allow to apply the argument in [19] and in [26]. This weight lets us to close the estimate in a weaker form than those obtained in [19] and [26], involving the lim inf instead of the lim.

Remark 1.4. Theorem 1.1 affirms that there exists a sequence of times $\left\{t_{n}: n \in \mathbb{Z}^{+}\right\}$with $t_{n} \uparrow \infty$ as $n \uparrow \infty$ such that

$$
\lim _{n \uparrow \infty} \int_{|x| \leq \frac{C t_{n}^{b}}{\log \left(t_{n}\right)}}\left(u^{2}+\left(q\left(\partial_{x}\right) u\right)^{2}\right)\left(x, t_{n}\right) d x=0 .
$$

Remark 1.5. It is known that the generalized $\mathrm{KdV}$ equation

$$
\partial_{t} u+\partial_{x}^{3} u+\partial_{x}(g(u))=0, \quad(t, x) \in \mathbb{R} \times \mathbb{R},
$$

possesses breather solutions, i.e. localized solutions which are also periodic in time, for $g(u)=u^{3}$ (modified KdV) and for $g(u)=u^{2}+\mu u^{3}$ (Gardner equation) with $\mu>0$, see [26]. In the case of the modified $\mathrm{KdV}$ these breather solutions can have arbitrarily small energy i.e. $H^{1}$-norm.

As in the case of the generalized BO equation, for the generalized ILW equations the energy is related to the $H^{1 / 2}$-norm. 
Next, we consider the initial value problem for the generalized Benjamin-Ono (gBO) equation

$$
\left\{\begin{array}{l}
\partial_{t} u+\mathcal{H} \partial_{x}^{2} u+\partial_{x}\left(u^{k}+p_{k}(u)\right)=0, \quad(t, x) \in \mathbb{R} \times \mathbb{R}, \quad k=2,3,4, \ldots \\
u(x, 0)=0
\end{array}\right.
$$

where

$$
p_{k}(s)=\sum_{j=k+1}^{m} a_{j} s^{j}, \quad m \in \mathbb{Z}^{+}, \quad a_{j} \in \mathbb{R}, \quad k=2,3, \ldots
$$

The following results are concerned with the non-existence of time periodic solutions to the IVP associated to the gBO equation (1.22):

Theorem 1.2. Let

$$
f(u):=u^{k}+p_{k}(u)
$$

with $k$ and $p_{k}(\cdot)$ as above. If a real non-trivial solution $u=u(t, x)$ of the IVP $(1.22)$

$$
u \in C\left(\mathbb{R}: H^{4}(\mathbb{R}) \cap L^{2}\left(|x|^{4} d x\right)\right) \cap C^{1}\left(\mathbb{R}: H^{2}(\mathbb{R}) \cap L^{2}\left(|x|^{2} d x\right)\right)
$$

is periodic in time with period $\omega$, then

$$
\int_{0}^{\omega} \int_{-\infty}^{\infty} f(u(t, x)) d x d t=0
$$

In particular, if

$$
\left\{\begin{array}{l}
f(u)=u^{2 j}, \quad j \in \mathbb{Z}^{+}, \\
\text {or } \\
f(u)=u^{2 j} \pm \rho u^{2 j+1}+\epsilon^{2} u^{2 j+2}, \quad 0 \leq \rho<2 \epsilon, \quad j \in \mathbb{Z}^{+},
\end{array}\right.
$$

then $u=u(t, x)$ cannot be time periodic.

Theorem 1.3. For any integer $k \geq 3$ and any polynomial $p_{k}(\cdot)$ in (1.23) there exists $\epsilon=\epsilon\left(k ; p_{k}\right)>0$ such that any real solution $u=u(t, x)$ of the IVP (1.22)

$$
u \in C\left(\mathbb{R}: H^{4}(\mathbb{R}) \cap L^{2}\left(|x|^{4} d x\right)\right) \cap C^{1}\left(\mathbb{R}: H^{2}(\mathbb{R}) \cap L^{2}\left(|x|^{2} d x\right)\right)
$$

corresponding to an initial data $u_{0}$ with

$$
0 \neq\left\|u_{0}\right\|_{1 / 2,2}<\epsilon
$$

cannot be time periodic.

Remark 1.6. (i) Real solution of the IVP associated to the equation (1.22) satisfy at least three conservation laws

$$
\begin{aligned}
& \mathcal{I}_{1}(u):=\int u(t, x) d x=\mathcal{I}_{1}\left(u_{0}\right), \quad \mathcal{I}_{2}(u):=\int u^{2}(t, x) d x=\mathcal{I}_{2}\left(u_{0}\right), \\
& \mathcal{I}_{3}(u):=\int_{-\infty}^{\infty}\left(\frac{\left(D_{x}^{1 / 2} u\right)^{2}}{2}-\left(\frac{u^{k+1}}{k+1}+G_{k}(u)\right)\right) d x=\mathcal{I}_{3}\left(u_{0}\right)
\end{aligned}
$$


where

$$
G_{k}(s)=\int_{0}^{s} p_{k}(r) d r
$$

(ii) From the conservation laws we have control of the $L^{p}$-norm of the small solution for $p \in[2, \infty)$ but not for $p=\infty$. This is the reason to consider only polynomial perturbations in (1.22) and (1.23).

(iii) The assumption (1.25) and (1.28), which justify the integrations by part in the proof, is not optimal.

Consider now that IVP associated to the generalized ILW (gILW) quation

$$
\left\{\begin{array}{l}
\partial_{t} u+\mathcal{T}_{\delta} \partial_{x}^{2} u+\frac{1}{\delta} \partial_{x} u+\partial_{x}\left(u^{k}+p_{k}(u)\right)=0, \quad(t, x) \in \mathbb{R} \times \mathbb{R}, \quad k=2,3,4, . . \\
u(x, 0)=u_{0}(x),
\end{array}\right.
$$

with $p_{k}(\cdot)$ as in (1.23). Similarly, to the results in Theorem 1.2 for the gBO equation for the gILW (1.32) we have:

Theorem 1.4. Let

$$
f(u):=u^{k}+p_{k}(u)
$$

with $k$ and $p_{k}(\cdot)$ as in (1.23). If a real non-trivial solution $u=u(t, x)$ of the IVP (1.32)

$$
u \in C\left(\mathbb{R}: H^{4}(\mathbb{R}) \cap L^{2}\left(|x|^{4} d x\right)\right) \cap C^{1}\left(\mathbb{R}: H^{2}(\mathbb{R}) \cap L^{2}\left(|x|^{2} d x\right)\right)
$$

is periodic in time with period $\omega$, then

$$
\int_{0}^{\omega} \int_{-\infty}^{\infty} f(u(t, x)) d x d t=0 .
$$

In particular, if

$$
\left\{\begin{array}{l}
f(u)=u^{2 j}, \quad j \in \mathbb{Z}^{+}, \\
\text {or } \\
f(u)=u^{2 j} \pm \rho u^{2 j+1}+\epsilon^{2} u^{2 j+2}, \quad 0 \leq \rho<2 \epsilon, \quad j \in \mathbb{Z}^{+},
\end{array}\right.
$$

then $u=u(t, x)$ cannot be time periodic.

From the arguments found in the proof of Theorem 1.1 one has that the proof of Theorem 1.4 becomes similar to that for Theorem 1.2. hence it will be omitted.

Now, we shall see that the propagation of regularity established in [8] and [9] in solutions of the $\mathrm{KdV}$ equations and $\mathrm{BO}$ equations respectively also holds in solutions of the ILW equation (1.1).

Theorem 1.5. Let $u_{0} \in H^{s}(\mathbb{R}), s>3 / 2$ and

$$
u \in C\left(\mathbb{R}: H^{s}(\mathbb{R})\right) \cap C^{1}\left([0, T]: H^{s-2}(\mathbb{R})\right) \cap L^{\infty}\left([0, T]: H_{l o c}^{s+1 / 2}(\mathbb{R})\right),
$$


be the corresponding solution of the IVP associated to the ILW equation (1.1) with data $u_{0}$ provided by Theorem (1). If for some $x_{0} \in \mathbb{R}, m \in \mathbb{Z}^{+}$and $m>s$ one has that

$$
M_{0}:=\int_{x_{0}}^{\infty}\left(\partial_{x}^{m} u_{0}(x)\right)^{2} d x<\infty
$$

then for any $\gamma>0, T>0, \epsilon>0, R>5 \epsilon$ it follows that

$$
\begin{aligned}
& \sup _{0 \leq t \leq T} \int_{x_{0}+\epsilon-\gamma t}\left(\partial_{x}^{m} u(t, x)\right)^{2} d x+\int_{0}^{T} \int_{x_{0}+\epsilon-\gamma t}^{x_{0}+R-\gamma t}\left(D_{x}^{1 / 2} \partial_{x}^{m} u(t, x)\right)^{2} d x d t \\
& \leq c=c\left(\left\|u_{0}\right\|_{s, 2} ; T ; M_{0} ; \gamma ; \epsilon ; R\right) .
\end{aligned}
$$

Our scheme to prove Theorem 1.5 will be to reduce it to the proof provided in [9] for solutions of the $\mathrm{BO}$ equation.

Our last theorem concerns with the far field region dominated by linear decay. A direct plane wave analysis of (1.1) reveals that, formally, linear waves tends to travel with nonnegative speeds, similar to $\mathrm{KdV}$ and $\mathrm{BO}$, and they concentrate in the spatial region $\{x \leq 0\}$. Here we prove that, despite the size of the data, the region of truly influence of linear waves is just $\left\{-t \log ^{1+\epsilon} t \ll x \leq 0\right\}$, for $\epsilon>0$.

Theorem 1.6. Assume $u \in C\left(\mathbb{R}: H^{1 / 2+}(\mathbb{R})\right) \cap L^{\infty}\left(\mathbb{R}: H^{1 / 2+}(\mathbb{R})\right)$. Then for any $\mu(t) \gtrsim$ $t \log ^{1+\epsilon} t$ and $\epsilon>0$,

$$
\lim _{t \rightarrow+\infty}\|u(t)\|_{L^{2}(|x| \sim \mu(t))}=0 .
$$

Remark 1.7. From the proof of Theorem 1.6, it will become clear that it is also valid for $\mathrm{KdV}, \mathrm{mKdV}, \mathrm{BBM}$ and $\mathrm{BO}$ equations. Therefore, this estimate is independent of the integrability of the equation. Note also that no size restriction is needed in (1.39), therefore it is a truly nonlinear decay estimate. It can be also rephrased as complete integrability cannot travel/move faster than $t \log t$.

Remark 1.8. Theorem 1.6 seems to us the first decay result in the energy space inside the linearly dispersive regime using nonintegrable techniques, with data only in the energy space.

An interesting by-product of Theorem 1.6 is the following result, in principle awkward in nature, that essentially says that integrability in time does not necessarily imply decay to zero in time.

Corollary 1.7. Under the hypothesis of Theorem 1.6, for any $\epsilon>0$ and any $\tilde{\phi} \epsilon$ $C_{0}^{\infty}([0,1]), \tilde{\phi}>0$ in $(0,1)$, one has

$$
\int_{10}^{\infty} \frac{1}{t}\left(\int_{-t}^{t \log ^{1+\epsilon} t-t}\left(\frac{x+t}{t \log ^{1+\epsilon} t}\right) \tilde{\phi}\left(\frac{x+t}{t \log ^{1+\epsilon} t}\right) u^{2}(t, x) d x\right) d t<+\infty .
$$


We emphasize that Corollary 1.7 is against intuition, because it shows integrability in time of a portion of the $L^{2}$ norm on a region where we know that solitons appear. For instance, (1.40) must hold for any fixed soliton $Q_{\delta, c}$ described in (1.11), and it is indeed the case. See Remark 5.1 for more details.

The rest of this paper is organized as follows. In section 2 we shall prove Theorem 1.1. Section 3 contains the proof of Theorem 1.2 and Theorem 1.3, Section 4 that of Theorem 1.5, and Section 5 the proof of Theorem 1.6 and Corollary 1.7.

Acknowledgments. We would like to thank C. Kwak for interesting comments and discussions about this work.

\section{Proof of Theorem 1.1}

First, we shall obtain some estimates concerning the symbol modeling the dispersive relation in the ILW equation (1.1)

$$
\sigma\left(\mathcal{T}_{\delta} \partial_{x}^{2}+\frac{\partial_{x}}{\delta}\right)(\xi)=-i\left((2 \pi \xi)^{2} \operatorname{coth}(2 \pi \delta \xi)-\frac{2 \pi \xi}{\delta}\right)=-i \Omega_{\delta}(2 \pi \xi)
$$

with

$$
\mathcal{T}_{\delta} \partial_{x}^{2}+\frac{\partial_{x}}{\delta}=-\mathcal{L}_{\delta}\left(\partial_{x}\right) \partial_{x}
$$

We observe that $\Omega_{\delta}(\cdot)$ is an smooth (analytic), real valued odd function with $\Omega_{\delta}(\xi)>0$ for $\xi>0$,

and

$$
\Omega_{\delta}(\xi)=\frac{1}{3} \frac{\xi^{3}}{\delta}+O\left(\frac{\xi^{5}}{\delta^{3}}\right) \quad \text { as } \quad|\xi| \downarrow 0
$$

$$
\Omega_{\delta}(\xi)=\xi|\xi|-\frac{\xi}{\delta}+O(1) \quad \text { as } \quad|\xi| \uparrow \infty .
$$

Moreover,

$$
\Omega_{\delta}^{\prime}(\xi)=\frac{\xi^{2}}{\delta}+O\left(\frac{\xi^{4}}{\delta^{3}}\right) \quad \text { as } \quad|\xi| \downarrow 0
$$

and

$$
\Omega_{\delta}^{\prime}(\xi)=2|\xi|-\frac{1}{\delta}+O\left(\frac{1}{\delta \xi}\right) \quad \text { as } \quad|\xi| \uparrow \infty .
$$

Also, $\Omega_{\delta}^{\prime}(\cdot)$ is even with $\Omega_{\delta}^{\prime}(0)=\Omega_{\delta}^{\prime \prime}(0)=0, \Omega_{\delta}^{\prime}(\xi)>0$ for $\xi>0$, and

$$
\left\|\Omega_{\delta}^{\prime \prime}\right\|_{\infty} \leq c=c(\delta) \text {. }
$$

Consequently, the symbol $\mathcal{L}_{\delta}(\xi)=\xi \operatorname{coth}(\delta \xi)-1$ of the operator $\mathcal{L}_{\delta}\left(\partial_{x}\right)$ in $(2.2)$ is smooth, even, positive for $\xi \neq 0$ with

$$
\mathcal{L}_{\delta}(0)=\mathcal{L}_{\delta}^{\prime}(0)=0 \quad \text { and } \quad \mathcal{L}_{\delta}^{\prime \prime}(0)=\frac{2}{3 \delta},
$$

and

$$
\mathcal{L}_{\delta}(\xi)=\frac{\xi^{2}}{3 \delta}+O(1) \quad \text { as } \quad \xi \uparrow \infty
$$


Next, we define the operator $q_{\delta}\left(\partial_{x}\right)$ whose symbol $q_{\delta}(\xi)$ is the square root of $\Omega_{\delta}^{\prime}(\xi)$, i.e.

$$
\Omega_{\delta}^{\prime}(\xi)=q_{\delta}(\xi) q_{\delta}(\xi)
$$

such that $q_{\delta}(\cdot)$ is even, $q_{\delta}(\xi)>0$ for $\xi>0$. Hence,

$$
q_{\delta}(\xi)=\frac{\xi}{\delta^{1 / 2}}+O\left(\frac{\xi^{3}}{\delta^{5 / 2}}\right) \quad \text { as } \quad \xi \downarrow 0
$$

and

$$
q_{\delta}(\xi)=\sqrt{\xi}\left(1-\frac{1}{2 \delta \xi}+O\left(\delta \xi^{-2}\right)\right) \quad \text { as } \quad \xi \uparrow \infty
$$

with

$$
q_{\delta}(0)=0 \quad \text { and } \quad q_{\delta}^{\prime}\left(0^{+}\right)=\frac{1}{\delta^{1 / 2}}
$$

In addition, we shall use that

$$
\mathcal{T}_{\delta} \partial_{x}^{2}+\frac{\partial_{x}}{\delta}=\partial_{x}^{2} \Psi_{\delta}\left(\partial_{x}\right)
$$

where the symbol of $i \Psi_{\delta}(\cdot), i \Psi(\xi)=1 / \xi-\operatorname{coth}(\delta \xi)$, is $C(\mathbb{R}) \cap L^{\infty}(\mathbb{R})$, odd, real valued and of order zero.

Without loss of generality, we assume now that $t \geq 10$ in (1.14). We recall that $a+b=$ $1, a, b \geq 0$. Let

$$
\lambda(t):=\frac{t^{b}}{\log t}, \quad \mu(t):=t^{a} \log ^{2} t
$$

Note that

$$
\frac{\lambda^{\prime}(t)}{\lambda(t)} \sim \frac{\mu^{\prime}(t)}{\mu(t)} \sim \frac{1}{t}, \quad \lambda(t) \mu(t)=t^{a+b} \log t
$$

This follows from

$$
\lambda^{\prime}(t)=\frac{b}{t^{1-b} \log t}-\frac{1}{t^{b} \log ^{2} t}=\frac{1}{t^{1-b} \log t}\left(b-\frac{1}{\log t}\right),
$$

and

$$
\frac{\lambda^{\prime}(t)}{\lambda(t)}=\frac{1}{t}\left(\frac{1}{2}-\frac{1}{\log t}\right), \quad \lambda^{\prime 2}(t)=\frac{1}{t \log ^{2} t}\left(\frac{1}{2}-\frac{1}{\log t}\right)^{2} .
$$

We introduce the following class of weight

$$
\begin{array}{r}
\mathcal{A}_{C}=\left\{\phi: \mathbb{R} \rightarrow \mathbb{R}: \quad \phi \text { smooth, } \phi(-C-1)=0, \operatorname{supp} \phi^{\prime} \subset[-C-1, C+1],\right. \\
\left.\phi^{\prime} \text { even, } \phi^{\prime}(x)=1 \text { for }|x| \leq C, \phi^{\prime}(x) \leq 0 \text { for } x \geq 0\right\} .
\end{array}
$$

We shall use that if $\phi \in \mathcal{A}_{C}$, then there exists $\phi_{0} \in \mathcal{A}_{C}$ such that

$$
\phi^{\prime}(x)=\left(\phi_{0}^{\prime}(x)\right)^{3} \text {. }
$$

This choice of weights is not essential but it will simplify the exposition. For each $\phi \in \mathcal{A}_{C}$ we consider the functional $\mathcal{V}(t)=\mathcal{V}_{\phi}(t)$ for $u$ solving $(1.1)$

$$
\mathcal{V}(t):=\frac{1}{\mu(t)} \int \phi\left(\frac{x}{\lambda(t)}\right) u(t, x) d x \text {. }
$$


Notice that from the hypothesis (1.17) of Theorem 1.1, one has

$$
\sup _{t \in \mathbb{R}} \mathcal{V}(t)<+\infty
$$

Lemma 2.1. We have

$$
\begin{aligned}
\frac{d}{d t} \mathcal{V}(t)= & -\frac{\lambda^{\prime}(t)}{\mu(t) \lambda(t)} \int \frac{x}{\lambda(t)} \phi^{\prime}\left(\frac{x}{\lambda(t)}\right) u(t, x) d x \\
& -\frac{\mu^{\prime}(t)}{\mu^{2}(t)} \int \phi\left(\frac{x}{\lambda(t)}\right) u(t, x) d x \\
& -\frac{1}{\mu(t) \lambda^{2}(t)} \int \phi^{\prime \prime}\left(\frac{x}{\lambda(t)}\right) \Psi_{\delta}\left(\partial_{x}\right) u(t, x) d x \\
& +\frac{1}{2 \mu(t) \lambda(t)} \int \phi^{\prime}\left(\frac{x}{\lambda(t)}\right) u^{2}(t, x) d x
\end{aligned}
$$

Proof. We have, using (2.14), that

$$
\begin{aligned}
\frac{d}{d t} \mathcal{V}(t)= & -\frac{\lambda^{\prime}(t)}{\mu(t) \lambda(t)} \int \frac{x}{\lambda(t)} \phi^{\prime}\left(\frac{x}{\lambda(t)}\right) u(t, x) d x-\frac{\mu^{\prime}(t)}{\mu^{2}(t)} \int \phi\left(\frac{x}{\lambda(t)}\right) u(t, x) d x \\
& +\frac{1}{\mu(t)} \int \phi\left(\frac{x}{\lambda(t)}\right) \partial_{t} u(t, x) d x \\
= & -\frac{\lambda^{\prime}(t)}{\mu(t) \lambda(t)} \int \frac{x}{\lambda(t)} \phi^{\prime}\left(\frac{x}{\lambda(t)}\right) u(t, x) d x-\frac{\mu^{\prime}(t)}{\mu^{2}(t)} \int \phi\left(\frac{x}{\lambda(t)}\right) u(t, x) d x \\
& -\frac{1}{\mu(t) \lambda(t)} \int \phi\left(\frac{x}{\lambda(t)}\right) \Psi_{\delta}\left(\partial_{x}\right) \partial_{x}^{2} u(t, x) d x+\frac{1}{2 \mu(t) \lambda(t)} \int \phi^{\prime}\left(\frac{x}{\lambda(t)}\right) u^{2}(t, x) d x \\
= & -\frac{\lambda^{\prime}(t)}{\mu(t) \lambda(t)} \int \frac{x}{\lambda(t)} \phi^{\prime}\left(\frac{x}{\lambda(t)}\right) u(t, x) d x-\frac{\mu^{\prime}(t)}{\mu^{2}(t)} \int \phi\left(\frac{x}{\lambda(t)}\right) u(t, x) d x \\
& -\frac{1}{\mu(t) \lambda^{2}(t)} \int \phi^{\prime \prime}\left(\frac{x}{\lambda(t)}\right) \Psi_{\delta}\left(\partial_{x}\right) u(t, x) d x \\
& +\frac{1}{2 \mu(t) \lambda(t)} \int \phi^{\prime}\left(\frac{x}{\lambda(t)}\right) u^{2}(t, x) d x .
\end{aligned}
$$

Combining the fact that $\Psi_{\delta}$ is a $C^{1}$ symbol of order zero with the second conservation law in (1.8) one gets that

$$
\left|\frac{1}{\mu(t) \lambda^{2}(t)} \int \phi^{\prime \prime}\left(\frac{x}{\lambda(t)}\right) \Psi_{\delta}\left(\partial_{x}\right) u(t, x) d x\right| \leq \frac{c}{\mu(t) \lambda^{3 / 2}}=\frac{c}{t^{a+3 b / 2}(\log (t))^{1 / 2}} .
$$

From the hypothesis (1.17) and the fact that

$$
\frac{x}{\lambda(t)} \phi^{\prime}\left(\frac{x}{\lambda(t)}\right) \in L^{\infty}(\mathbb{R}) \quad \text { uniformly in } \quad t \in[10, \infty)
$$


we have

$$
\left|\frac{\lambda^{\prime}(t)}{\mu(t) \lambda(t)} \int \frac{x}{\lambda(t)} \phi^{\prime}\left(\frac{x}{\lambda(t)}\right) u(t, x) d x\right| \lesssim \frac{1}{t \log ^{2} t}
$$

Similarly,

$$
\left|\frac{\mu^{\prime}(t)}{\mu^{2}(t)} \int \phi\left(\frac{x}{\lambda(t)}\right) u(t, x) d x\right| \lesssim \frac{1}{t \log ^{2} t}
$$

Inserting the above estimates in (2.21) it follows that

$$
\frac{d}{d t} \mathcal{V}(t)=\frac{1}{2 \mu(t) \lambda(t)} \int \phi^{\prime}\left(\frac{x}{\lambda(t)}\right) u^{2}(t, x) d x+h(t)
$$

with $h \in L^{1}([10, \infty))$. From this estimate, we clearly see that

$$
\int_{10}^{\infty} \frac{1}{\mu(t) \lambda(t)} \int \phi^{\prime}\left(\frac{x}{\lambda(t)}\right) u^{2}(t, x) d x d t=c_{\phi}<+\infty .
$$

for any weight $\phi(\cdot)$ in the class $\mathcal{A}$ defined in (2.17) and since $\frac{1}{\mu(t) \lambda(t)}$ does not integrate in $[10, \infty)$, it follows that for some increasing sequence of time $t_{n} \rightarrow+\infty$,

$$
\lim _{n \rightarrow \infty} \int_{-\infty}^{\infty} \phi\left(\frac{x}{\lambda\left(t_{n}\right)}\right) u^{2}\left(t_{n}, x\right) d x=0 .
$$

Next, we define the functional $\mathcal{J}(t)$ as

$$
\mathcal{J}(t):=\frac{1}{2 \mu(t)} \int \phi\left(\frac{x}{\lambda(t)}\right) u^{2}(t, x) d x .
$$

We claim the following result.

Lemma 2.2. We have

$$
\begin{aligned}
\frac{d}{d t} \mathcal{J}(t)= & -\frac{\mu^{\prime}(t)}{2 \mu^{2}(t)} \int \phi\left(\frac{x}{\lambda(t)}\right) u^{2}(t, x) d x-\frac{\lambda^{\prime}(t)}{2 \mu(t) \lambda(t)} \int \frac{x}{\lambda(t)} \phi^{\prime}\left(\frac{x}{\lambda(t)}\right) u^{2}(t, x) d x \\
& +\frac{1}{\mu(t)} \int \frac{1}{\lambda(t)} \phi\left(\frac{x}{\lambda(t)}\right) u\left(-\mathcal{T}_{\delta} \partial_{x}^{2}-\frac{\partial_{x}}{\delta}\right) u(t, x) d x \\
& +\frac{1}{3 \lambda(t) \mu(t)} \int \phi^{\prime}\left(\frac{x}{\lambda(t)}\right) u^{3}(t, x) d x .
\end{aligned}
$$


Proof. We have, using (2.2), that

$$
\begin{aligned}
\frac{d}{d t} \mathcal{J}(t)= & -\frac{\mu^{\prime}(t)}{2 \mu^{2}(t)} \int \phi\left(\frac{x}{\lambda(t)}\right) u^{2}(t, x) d x-\frac{\lambda^{\prime}(t)}{2 \mu(t) \lambda(t)} \int \frac{x}{\lambda(t)} \phi^{\prime}\left(\frac{x}{\lambda(t)}\right) u^{2}(t, x) d x \\
& +\frac{1}{\mu(t)} \int \phi\left(\frac{x}{\lambda(t)}\right)\left(u \partial_{t} u\right)(t, x) d x \\
= & -\frac{\mu^{\prime}(t)}{2 \mu^{2}(t)} \int \phi\left(\frac{x}{\lambda(t)}\right) u^{2}(t, x) d x-\frac{\lambda^{\prime}(t)}{2 \mu(t) \lambda(t)} \int \frac{x}{\lambda(t)} \phi^{\prime}\left(\frac{x}{\lambda(t)}\right) u^{2}(t, x) d x \\
& +\frac{1}{\mu(t)} \int \phi\left(\frac{x}{\lambda(t)}\right) u\left(-\mathcal{T}_{\delta} \partial_{x}^{2} u-\frac{\partial_{x} u}{\delta}-u \partial_{x} u\right)(t, x) d x \\
= & -\frac{\mu^{\prime}(t)}{2 \mu^{2}(t)} \int \phi\left(\frac{x}{\lambda(t)}\right) u^{2}(t, x) d x-\frac{\lambda^{\prime}(t)}{2 \mu(t) \lambda(t)} \int \frac{x}{\lambda(t)} \phi^{\prime}\left(\frac{x}{\lambda(t)}\right) u^{2}(t, x) d x \\
& -\frac{1}{\mu(t)} \int \phi\left(\frac{x}{\lambda(t)}\right) u(t, x) \partial_{x} \mathcal{L}_{\delta}\left(\partial_{x}\right) u(t, x) d x \\
& +\frac{1}{3 \lambda(t) \mu(t)} \int \phi^{\prime}\left(\frac{x}{\lambda(t)}\right) u^{3}(t, x) d x .
\end{aligned}
$$

The proof is complete.

Next, we procede to bound the terms appearing in Lemma 2.2 in the right hand side of (2.27). First, from (2.15) we have

$$
\left|-\frac{\mu^{\prime}(t)}{2 \mu^{2}(t)} \int \phi\left(\frac{x}{\lambda(t)}\right) u^{2}(t, x) d x\right| \lesssim \frac{1}{t^{a+1} \log ^{2} t},
$$

which is integrable in $[10, \infty)$. Next, we have again the bound

$$
\left|\frac{\lambda^{\prime}(t)}{2 \mu(t) \lambda(t)} \int \frac{x}{\lambda(t)} \phi^{\prime}\left(\frac{x}{\lambda(t)}\right) u^{2}(t, x) d x\right| \lesssim \frac{1}{t^{a+1} \log ^{2} t} .
$$

Now we deal with the term

$$
A_{1}(t):=\frac{1}{\mu(t)} \int \phi\left(\frac{x}{\lambda(t)}\right) u(t, x) \mathcal{L}_{\delta}\left(\partial_{x}\right) \partial_{x} u(t, x) d x,
$$

which requires more care. Since the symbol $\mathcal{L}_{\delta}\left(\partial_{x}\right)$ is even one has that

$$
\begin{aligned}
A_{1}(t) & =-\frac{1}{\mu(t)} \int \mathcal{L}_{\delta}\left(\partial_{x}\right) \partial_{x}\left(\phi\left(\frac{x}{\lambda(t)}\right) u(t, x)\right) u(t, x) d x \\
& =-\frac{1}{\mu(t)} \int \phi\left(\frac{x}{\lambda(t)}\right) u(t, x) \mathcal{L}_{\delta}\left(\partial_{x}\right) \partial_{x} u(t, x) d x \\
& -\frac{1}{\mu(t)} \int u(t, x)\left[\mathcal{L}_{\delta}\left(\partial_{x}\right) \partial_{x} ; \phi\left(\frac{x}{\lambda(t)}\right)\right] u(t, x) d x .
\end{aligned}
$$

Therefore,

$$
2 A_{1}(t)=-\frac{1}{\mu(t)} \int u(t, x)\left[\mathcal{L}_{\delta}\left(\partial_{x}\right) \partial_{x} ; \phi\left(\frac{x}{\lambda(t)}\right)\right] u(t, x) d x
$$


Using Fourier transform one sees that

$$
\begin{aligned}
& \left(\left[\mathcal{L}_{\delta}\left(\widehat{\left.\partial_{x}\right) \partial_{x}} ; \phi\right] f\right)(\xi)\right. \\
& =c \int\left(\Omega_{\delta}(\xi)-\Omega_{\delta}(\eta)\right) \widehat{\phi}(\xi-\eta) \widehat{f}(\eta) d \eta
\end{aligned}
$$

Since

$$
\Omega_{\delta}(\xi)-\Omega_{\delta}(\eta)=(\xi-\eta) \Omega_{\delta}^{\prime}(\eta)+\frac{(\xi-\eta)^{2}}{2} \Omega_{\delta}^{\prime \prime}(\theta \eta+(1-\theta) \xi)
$$

with $\theta=\theta(\xi, \eta) \in[0,1]$ and $\left\|\Omega_{\delta}^{\prime \prime}\right\|_{\infty} \leq c_{\delta}$ it follows that

$$
\left[\mathcal{L}_{\delta}\left(\partial_{x}\right) \partial_{x} ; \phi\right] f(x)=c \phi^{\prime}(x)\left(\Omega_{\delta}^{\prime}\left(\partial_{x}\right) f\right)(x)+R_{1}(x),
$$

with the term $R_{1}$ (after combining Plancherel identity, Minkowski integral inequality and Sobolev embedding) satisfying the estimate

$$
\left\|R_{1}\right\|_{2} \leq\left\|\widehat{\phi^{\prime \prime}}\right\|_{1}\|f\|_{2} \leq c\left\|\phi^{\prime \prime}\right\|_{1,2}\|f\|_{2}
$$

where $\|h\|_{k, 2}=\left\|\left(1-\partial_{x}^{2}\right)^{k / 2} h\right\|_{2}, \quad k \in \mathbb{R}$.

Inserting this estimate in (2.30) we can conclude that

$$
A_{1}(t)=\frac{c}{\mu(t) \lambda(t)} \int u(t, x) \phi^{\prime}\left(\frac{x}{\lambda(t)}\right) \Omega_{\delta}^{\prime}\left(\partial_{x}\right) u(t, x) d x+r_{1}(t)
$$

with $r_{1} \in L^{1}([10, \infty))$.

From (2.35) we can write, using that the symbol of $q\left(\partial_{x}\right)$ is even (2.10) that

$$
\begin{aligned}
\mu(t) \lambda(t)\left(A_{1}(t)+r_{1}(t)\right)= & c \int \phi^{\prime}\left(\frac{x}{\lambda(t)}\right) u(t, x) q\left(\partial_{x}\right) q\left(\partial_{x}\right) u(t, x) d x \\
= & c \int q\left(\partial_{x}\right)\left(\phi^{\prime}\left(\frac{x}{\lambda(t)}\right) u(t, x)\right) q\left(\partial_{x}\right) u(t, x) d x \\
= & c \int \phi^{\prime}\left(\frac{x}{\lambda(t)}\right) q\left(\partial_{x}\right) u(t, x) q\left(\partial_{x}\right) u(t, x) d x \\
& +c \int q\left(\partial_{x}\right)\left[q\left(\partial_{x}\right) ; \phi^{\prime}\left(\frac{\cdot}{\lambda(t)}\right)\right] u(t, x) u(t, x) d x
\end{aligned}
$$

We claim :

$$
\left\|q\left(\partial_{x}\right)\left[q\left(\partial_{x}\right) ; \rho\right] f\right\|_{2} \leq c\left\|\partial_{x} \rho\right\|_{2,2}\|f\|_{2} .
$$

To prove (2.37) we take Fourier transform to get

$$
\left.\left(q\left(\partial_{x}\right) \widehat{\left[q\left(\partial_{x}\right)\right.} ; \rho\right] f\right)(\xi)=c q(\xi) \int(q(\xi)-q(\eta)) \widehat{\rho}(\xi-\eta) \widehat{f}(\eta) d \eta
$$

We observe : if $\xi \eta \geq 0$, then

$$
|q(\xi)||q(\xi)-q(\eta)| \leq c|q(\xi)|\left|q^{\prime}(\theta \eta+(1-\theta) \xi)\right||\xi-\eta| \leq c|\xi-\eta|(1+|\xi-\eta|) .
$$


see (2.10)-(2.13), and if $\xi \eta<0$, then

$$
|q(\xi)||q(\xi)-q(\eta)| \leq c|\xi-\eta|(1+|\xi-\eta|) .
$$

Hence,

$$
\begin{aligned}
& \left\|q\left(\partial_{x}\right)\left[q\left(\partial_{x}\right) ; \rho\right] f\right\|_{2} \leq c \|\left(\left(1-\widehat{\left.\partial_{x}^{2}\right)^{1 / 2}} \partial_{x} \rho\right) * \widehat{f} \|_{2}\right. \\
& \leq c \|\left(\left(1-\widehat{\left.\partial_{x}^{2}\right)^{1 / 2}} \partial_{x} \rho\right)\left\|_{1}\right\| \widehat{f}\left\|_{2} \leq c\right\|\left(1-\partial_{x}^{2}\right)^{1 / 2} \partial_{x} \rho\left\|_{1,2}\right\| f \|_{2}\right. \\
& \leq c\left\|\partial_{x} \rho\right\|_{2,2}\|f\|_{2} .
\end{aligned}
$$

In our case $\rho(x)=\phi^{\prime}\left(\frac{x}{\lambda(t)}\right)$, therefore

$$
\left\|q\left(\partial_{x}\right)\left[q\left(\partial_{x}\right) ; \phi^{\prime}\left(\frac{\cdot}{\lambda(t)}\right)\right] u\right\|_{2} \leq \frac{c}{(\lambda(t))^{1 / 2}}\left\|\phi^{\prime \prime}\right\|_{1,2}\|u\|_{2},
$$

which inserted in (2.36) tells us that

$$
A_{1}(t)=\frac{1}{\mu(t) \lambda(t)} \int \phi^{\prime}\left(\frac{x}{\lambda(t)}\right) q\left(\partial_{x}\right) u q\left(\partial_{x}\right) u(t, x) d x+r_{1}(t)+g(t),
$$

with

$$
|g(t)| \leq \frac{c}{\mu(t)(\lambda(t))^{3 / 2}} \in L^{1}([10, \infty)) .
$$

Finally, we shall bound the last term in (2.27). Combining the remark in (2.18), the calculus for fractional derivatives deduce in [13] (Appendix) and the conservation laws in (1.8) we write

$$
\begin{aligned}
\mid \frac{1}{\lambda(t) \mu(t)} & \int \phi^{\prime}\left(\frac{x}{\lambda(t)}\right) u^{3}(t, x) d x \mid=\frac{1}{\lambda(t) \mu(t)}\left\|\phi_{0}^{\prime}\left(\frac{\cdot}{\lambda(t)}\right) u(t, \cdot)\right\|_{3}^{3} \\
& \leq c \frac{1}{\lambda(t) \mu(t)}\left\|D_{x}^{1 / 2}\left(\phi_{0}^{\prime}\left(\frac{\cdot}{\lambda(t)}\right) u(t, \cdot)\right)\right\|_{2}\left\|\phi_{0}^{\prime}\left(\frac{\cdot}{\lambda(t)}\right) u^{2}(t, \cdot)\right\|_{2}^{2} \\
& \leq c^{*} \frac{1}{\lambda(t) \mu(t)} \int \phi_{0}^{\prime}\left(\frac{x}{\lambda(t)}\right) u^{2}(t, x) d x .
\end{aligned}
$$

with $c^{*}=c^{*}\left(\left\|u_{0}\right\|_{1 / 2,2} ;\left\|\phi_{0}^{\prime}\right\|_{1,2}\right)$. Using (2.24) with $\phi_{0} \in \mathcal{A}_{C}$ instead of $\phi \in \mathcal{A}_{C}$ it follows that the last term in $(2.41)$ belongs to $L^{1}([10, \infty)$.

Now gathering the above results one has that

$$
\frac{d}{d t} \mathcal{J}(t)=\frac{1}{2 \mu(t) \lambda(t)} \int \phi^{\prime}\left(\frac{x}{\lambda(t)}\right)\left(q\left(\partial_{x}\right) u\right)^{2}(t, x) d x+h_{1}(t)
$$

with $h_{1} \in L^{1}([10, \infty))$. Therefore, from the conservation laws in (1.8) one concludes that

$$
\int_{10}^{\infty} \frac{1}{\mu(t) \lambda(t)} \int \phi^{\prime}\left(\frac{x}{\lambda(t)}\right)\left(q\left(\partial_{x}\right) u\right)^{2}(t, x) d x d t<+\infty .
$$

This combines with (2.24) and (2.11)-(2.13) leads us to the estimate

$$
\int_{10}^{\infty} \frac{1}{\mu(t) \lambda(t)} \int \phi^{\prime}\left(\frac{x}{\lambda(t)}\right)\left(\left(q\left(\partial_{x}\right) u\right)^{2}+u^{2}\right)(t, x) d x d t<+\infty
$$


which yields the desired result (1.18) and basically completes the proof of Theorem 1.1.

Remark 2.1. In [12] the estimate (2.41) was established for solutions of the BO equation by fixing the weight

$$
\phi(x)=\frac{\pi}{2}+\arctan (x) \quad \text { so that } \quad \phi^{\prime}(x)=\frac{1}{1+x^{2}},
$$

which resembles the profile of the soliton solution of the $\mathrm{BO}$ equation.

\section{Proof of Theorems 1.2-1.3}

Proof of Theorem 1.2. We observe that multiplying the equation in (1.22) by $x$ and integrating after some integration by part one obtains

$$
\frac{d}{d t} \int_{-\infty}^{\infty} x u(t, x) d x=\int_{-\infty}^{\infty} f(u(t, x)) d x
$$

which yields the desired result.

Proof of Theorem 1.3. Multiplying the equation (1.22) by $\mathcal{H} \partial_{x} u-\left(u^{k}+p_{k}(u)\right)$ and integration the result one gets

$$
\frac{d}{d t} \int_{-\infty}^{\infty}\left(\frac{\left(D_{x}^{1 / 2} u\right)^{2}}{2}-\left(\frac{u^{k+1}}{k+1}+G_{k}(u)\right)\right) d x=\frac{d}{d t} I_{3}(u)=0
$$

where

$$
G_{k}(s)=\int_{0}^{s} p_{k}(r) d r .
$$

Now multiplying the equation $(1.22)$ by $x u(t, x)$ we obtain

$$
\frac{1}{2} \frac{d}{d t} \int u^{2} x d x+\int \mathcal{H} \partial_{x}^{2} u u x d x+\int \partial_{x}\left(u^{k}+p_{k}(u)\right) u x d x=0 .
$$

First, we consider the second term in (3.4)

$$
\begin{aligned}
E_{1} & :=\int \mathcal{H} \partial_{x}^{2} u u x d x=-\int \mathcal{H} \partial_{x} u \partial_{x} u x d x-\int \mathcal{H} \partial_{x} u u d x \\
& =E_{1,1}-\int\left(D_{x}^{1 / 2} u\right)^{2} d x .
\end{aligned}
$$

We claim that

$$
E_{1,1}=-\int \mathcal{H} \partial_{x} u \partial_{x} u x d x=0
$$

By integration by part

$$
\begin{aligned}
E_{1,1} & =\int \partial_{x} u \mathcal{H}\left(\partial_{x} u x\right) d x \\
& =\int \partial_{x} u \mathcal{H} \partial_{x} u x d x+\int \partial_{x} u[\mathcal{H} ; x] \partial_{x} u d x .
\end{aligned}
$$


Therefore,

$$
E_{1,1}=-\frac{1}{2} \int u \partial_{x}[\mathcal{H} ; x] \partial_{x} u d x
$$

To obtain the claim it suffices to see that if

$$
\partial_{x}[\mathcal{H} ; x] \partial_{x} u \equiv 0,
$$

which follows the identities

$$
\left(\partial_{x}\left[\widehat{\mathcal{H} ; x]} \partial_{x} u\right)(\xi, t)=-2 \pi\left(|\xi| \partial_{\xi}(\xi \widehat{u}(\xi, t))-\xi \partial_{\xi}(|\xi| \widehat{u}(\xi, t))\right)=0 .\right.
$$

Returning to (3.4) we observe that

$$
\begin{aligned}
\int \partial_{x}\left(u^{k}+p_{k}(u)\right) u x d x & =\int \partial_{x}\left(\frac{k}{k+1} u^{k+1}+P_{k}(u)\right)(t, x) x d x \\
& =-\frac{k}{k+1} \int u^{k+1} d x-\int P_{k}(u) d x
\end{aligned}
$$

where $\partial_{x} P_{k}(s)=s \partial_{x} p_{k}(s)$.

Thus, inserting (3.5), (3.6), and (3.11) in (3.4) one gets that

$$
\frac{1}{2} \frac{d}{d t} \int u^{2}(t, x) x d x-\int\left(D_{x}^{1 / 2} u\right)^{2} d x-\frac{k}{k+1} \int u^{k+1} d x-\int P_{k}(u) d x=0
$$

which gives that

$$
\frac{1}{2} \frac{d}{d t} \int u^{2}(t, x) x d x=2 I_{3}\left(u_{0}\right)+\frac{k+2}{k+1} \int u^{k+1} d x+\int\left(2 G_{k}(u)+P_{k}(u)\right) d x .
$$

Next, we recall the following inequality of Gagliardo-Nirenberg type : for $q \in[2, \infty)$

$$
\|f\|_{q} \leq c_{q}\left\|D^{1 / 2-1 / q} f\right\|_{2} \leq c_{q}\|f\|_{2}^{2 / q}\left\|D^{1 / 2} f\right\|_{2}^{1-2 / q} .
$$

Using the notation

$$
y(t)=\left\|D_{x}^{1 / 2} u(t)\right\|_{2},
$$

and combining (3.2), (1.23) and (3.14) one sees that

$$
\frac{d}{d t} \int_{-\infty}^{\infty} x u(t, x) d x=\int_{-\infty}^{\infty} f(u(t, x)) d x .
$$

An integration in the time interval $[0, \omega]$ completes the proof.

\section{Proof of Theorem 1.5}

Proof. First, we consider the principal symbol of the operator modeling the dispersive relation in the ILW equation (1.1)

$$
\sigma\left(\mathcal{T}_{\delta} \partial_{x}^{2}\right)=-4 \pi^{2} \xi^{2} \operatorname{coth}(2 \pi \delta \xi) i
$$


Thus,

$$
\begin{aligned}
& 4 \pi^{2}|\xi| \xi-4 \pi^{2} \xi^{2} \operatorname{coth}(2 \pi \delta \xi) \\
& =4 \pi^{2}|\xi| \xi(1-\operatorname{sgn}(\xi) \operatorname{coth}(2 \pi \delta \xi)) \\
& =4 \pi^{2}|\xi| \xi\left(-\frac{2 e^{-4 \pi \delta|\xi|}}{1-e^{-4 \pi \delta|\xi|}}\right) .
\end{aligned}
$$

Hence, for any $M>0$ sufficiently large (such that $e^{-4 \pi^{2} \delta M} \leq 1 / 2$ ) one has

$$
\sup _{|\xi| \geq M}|| 4 \pi^{2}|\xi| \xi-4 \pi^{2} \xi^{2} \operatorname{coth}(2 \pi \delta \xi) \mid \leq 16 M^{2} e^{-4 \pi^{2} \delta M}
$$

Now, taking $\chi \in C_{0}^{\infty}(\mathbb{R})$ such that supp $\subset[-1,1]$ with $\chi(x)=1, x \in[-1 / 2,1 / 2]$ and $\chi(x) \geq 0, x \in \mathbb{R}$. one sees from (4.3) that for any $R>0$

$$
\sigma_{R, 1}(\xi)=\left(4 \pi^{2}|\xi| \xi-4 \pi^{2} \xi^{2} \operatorname{coth}(2 \pi \delta \xi)\right)(1-\chi(\xi / R)) \in S^{-\infty}
$$

i.e. $\sigma_{R, 1}$ is the symbol of a smoothing pseudo-differential operator and that for any $R>0$

$$
\sigma_{R, 2}(\xi)=\left(4 \pi^{2}|\xi| \xi-4 \pi^{2} \xi^{2} \operatorname{coth}(2 \pi \delta \xi)\right) \chi(x / R)
$$

is a multiplier with compact support.

Therefore, the operator $A_{\delta_{R}}\left(\partial_{x}\right)$ with symbol $\sigma\left(A_{\delta_{R}}\right)(\xi)=4 \pi^{2}|\xi| \xi-4 \pi^{2} \xi^{2} \operatorname{coth}(2 \pi \delta \xi)$ satisfies that for any $s \geq 0$

$$
\left\|A_{\sigma_{R}} f\right\|_{s, 2} \leq c\|f\|_{2}, \quad \text { with } \quad c=c(s ; \delta) .
$$

Thus, rewriting the ILW equation (1.1) as

$$
\partial_{t} u+\mathcal{H} \partial_{x}^{2} u+\left(\mathcal{T}_{\delta} \partial_{x}^{2}-\mathcal{H} \partial_{x}^{2}\right) u+\frac{1}{\delta} \partial_{x} u+u \partial_{x} u=0
$$

we observe that the argument carried out in [9] for the BO equation based on weighted energy estimates can be applied for the equation in (4.7) without any major modification.

This essentially yields the proof of Theorem 1.5.

\section{Proof of Theorem 1.6 And Corollary 1.7}

We follow the proof of Theorem 1.1 with some key differences. The most important is that now we choose $\lambda(t)$ such that $\lambda^{-1}(t)$ is integrable in time. Set now (compare with $(2.15))$, for $t \geq 10$ and $\epsilon>0$,

$$
\lambda(t):=t \log ^{1+\epsilon} t, \quad \mu(t):=\lambda(t)=t \log ^{1+\epsilon} t .
$$

Any other choice of $\lambda(t)$ (and therefore, $\mu(t)$ ) which is bigger in size, also works. Note that $1 / \lambda(t)$ is now integrable in $[10, \infty)$, and

$$
\frac{\lambda^{\prime}(t)}{\lambda(t)}=\frac{\mu^{\prime}(t)}{\mu(t)} \sim \frac{1}{t}, \quad \frac{\mu^{\prime}(t)}{\lambda(t)} \sim \frac{1}{t} .
$$

Recall that now $\frac{\mu^{\prime}(t)}{\lambda(t)}$ is not integrable in $[2, \infty)$. 
Now, as in (2.26), we define the modified functional $\mathcal{J}_{e}(t)$ as

$$
\mathcal{J}_{e}(t):=\frac{1}{2} \int \phi\left(\frac{x+\mu(t)}{\lambda(t)}\right) u^{2}(t, x) d x .
$$

Here, $\phi$ is a smooth bounded function to be chosen later. Following the lines of the proof of Lemma 2.2, we claim

Lemma 5.1. We have

$$
\begin{aligned}
\frac{d}{d t} \mathcal{J}_{e}(t)= & \frac{\mu^{\prime}(t)}{2 \lambda(t)} \int \phi^{\prime}\left(\frac{x+\mu(t)}{\lambda(t)}\right) u^{2}(t, x) d x \\
& -\frac{\lambda^{\prime}(t)}{2 \lambda(t)} \int\left(\frac{x+\mu(t)}{\lambda(t)}\right) \phi^{\prime}\left(\frac{x+\mu(t)}{\lambda(t)}\right) u^{2}(t, x) d x \\
& +\int \phi\left(\frac{x+\mu(t)}{\lambda(t)}\right) u\left(-\mathcal{T}_{\delta} \partial_{x}^{2}-\frac{\partial_{x}}{\delta}\right) u(t, x) d x \\
& +\frac{1}{3 \lambda(t)} \int \phi^{\prime}\left(\frac{x+\mu(t)}{\lambda(t)}\right) u^{3}(t, x) d x .
\end{aligned}
$$

Now, we prove decay on the left portion $x \sim-\lambda(t)$. First of all, choose $\phi \in C^{\infty} \cap L^{\infty}$ such that $\phi(x) \in[0,1]$ for all $x \in \mathbb{R}, \phi(s)=1$ if $s \leq-1, \phi(s)=0$ for $s \geq 0$, and $\phi^{\prime} \leq 0$ in $\mathbb{R}$, see (2.17).

Notice that $\phi^{\prime}\left(\frac{x+\mu(t)}{\lambda(t)}\right) \neq 0$ only in the region of $x \in \mathbb{R}$ such that $-1 \leq \frac{x+\mu(t)}{\lambda(t)} \leq 0$, essentially nonpositive. Therefore, for all $x \in \mathbb{R}$,

$$
\phi^{\prime}\left(\frac{x+\mu(t)}{\lambda(t)}\right) \leq 0, \quad\left(\frac{x+\mu(t)}{\lambda(t)}\right) \phi^{\prime}\left(\frac{x+\mu(t)}{\lambda(t)}\right) \geq 0 .
$$

Since

$$
\int \phi\left(\frac{x+\mu(t)}{\lambda(t)}\right) u\left(-\mathcal{T}_{\delta} \partial_{x}^{2}-\frac{\partial_{x}}{\delta}\right) u(t, x) d x=-\int \phi\left(\frac{x}{\lambda(t)}\right) u(t, x) \mathcal{L}_{\delta}\left(\partial_{x}\right) \partial_{x} u(t, x) d x,
$$

proceeding as in the estimate of (2.28), we get

$$
\left|\int \phi\left(\frac{x}{\lambda(t)}\right) u(t, x) \mathcal{L}_{\delta}\left(\partial_{x}\right) \partial_{x} u(t, x) d x\right| \lesssim \frac{1}{\lambda(t)}
$$

Now, the cubic term in (5.3) is treated in similar fashion as in (2.41). We obtain that

$$
\left|\frac{1}{3 \lambda(t)} \int \phi^{\prime}\left(\frac{x+\mu(t)}{\lambda(t)}\right) u^{3}(t, x) d x\right| \lesssim \frac{1}{\lambda(t)} .
$$

Consequently, following the proof of Theorem 1.1, and using that $\lambda^{-1}(t)$ integrates in time, we have from (5.3):

$$
\begin{aligned}
\int_{10}^{\infty} \frac{1}{t} & \left(\int-\phi^{\prime}\left(\frac{x+\mu(t)}{\lambda(t)}\right) u^{2}(t, x) d x\right. \\
& \left.+\int\left(\frac{x+\mu(t)}{\lambda(t)}\right) \phi^{\prime}\left(\frac{x+\mu(t)}{\lambda(t)}\right) u^{2}(t, x) d x\right) d t<+\infty .
\end{aligned}
$$


Hence, a sequence of times $t_{n} \uparrow+\infty$ is such that the integrand converges to zero. Once again in (5.3), choose now $\widetilde{\phi} \in C_{0}^{\infty}(\mathbb{R})$ such that

$$
\begin{aligned}
& \widetilde{\phi}(x) \in[0,1] \text { for all } x \in \mathbb{R}, \widetilde{\phi}(s)=0 \text { if } s \leq-3 / 4, \widetilde{\phi}(s)=1 \text { for } s \geq-1 / 4, \\
& \text { and } \widetilde{\phi^{\prime}} \geq 0 \text { in } \mathbb{R} \text {. }
\end{aligned}
$$

A new estimate of $\frac{d}{d t} \mathcal{J}_{e}(t)$ gives

$$
\begin{aligned}
\left|\frac{d}{d t} \mathcal{J}_{e}(t)\right| \lesssim & \frac{1}{t} \int \widetilde{\phi}^{\prime}\left(\frac{x+\mu(t)}{\lambda(t)}\right) u^{2}(t, x) d x \\
& +\frac{1}{t} \int\left(\frac{x+\mu(t)}{\lambda(t)}\right) \widetilde{\phi}^{\prime}\left(\frac{x+\mu(t)}{\lambda(t)}\right) u^{2}(t, x) d x+\frac{1}{t \log ^{1+\epsilon} t} \\
\lesssim & \frac{1}{t} \int \phi^{\prime}\left(\frac{x+\mu(t)}{\lambda(t)}\right) u^{2}(t, x) d x+\frac{1}{t \log ^{1+\epsilon} t} .
\end{aligned}
$$

The rest of the proof is direct, see e.g. [26]. The final conclusion follows from the fact that $-\frac{3}{4} \leq \frac{x+\mu(t)}{\lambda(t)} \leq-\frac{1}{4}$ is equivalent to $x \sim-\mu(t)=-\lambda(t)$.

5.1. Proof of Corollary 1.7. The proof is simple, just a modification of certain aspects of the proof of Theorem 1.6.

Set now, for $t \geq 10$, and $\varepsilon>0$,

$$
\lambda(t):=t \log ^{1+\epsilon} t, \quad \mu(t):=t .
$$

Note that $1 / \lambda(t)$ is now integrable in $[10, \infty)$, and

$$
\frac{\lambda^{\prime}(t)}{\lambda(t)}=\frac{\mu^{\prime}(t)}{\mu(t)} \sim \frac{1}{t}, \quad \frac{\mu^{\prime}(t)}{\lambda(t)} \sim \frac{1}{t \log ^{1+\epsilon} t} .
$$

Recall that now $\frac{\mu^{\prime}(t)}{\lambda(t)}$ is integrable in $[2, \infty)$.

Now we consider the same modified functional $\mathcal{J}_{e}(t)$ as in (5.2). Choose $\phi \in C^{\infty} \cap L^{\infty}$ such that $\phi(x) \in[0,1]$ for all $x \in \mathbb{R}, \phi(s)=0$ if $s \leq 0, \phi(s)=1$ for $s \geq 1$, and $\phi^{\prime} \geq 0$ in $\mathbb{R}$.

Notice that $\phi^{\prime}\left(\frac{x+\mu(t)}{\lambda(t)}\right) \neq 0$ only in the region of $x \in \mathbb{R}$ such that $0 \leq \frac{x+\mu(t)}{\lambda(t)} \leq 1$, essentially nonnegative. This region reads

$$
-t \leq x \leq t \log ^{1+\epsilon} t-t
$$

hence it contains the region where solitons exist. Therefore, for all $x \in \mathbb{R}$,

$$
\left(\frac{x+\mu(t)}{\lambda(t)}\right) \phi^{\prime}\left(\frac{x+\mu(t)}{\lambda(t)}\right) \geq 0 \text {. }
$$

Proceeding as in the proof of Theorem 1.6, we obtain now the weaker condition

$$
\int_{10}^{\infty} \frac{1}{t}\left(\int_{-t}^{t \log ^{1+\epsilon} t-t}\left(\frac{x+\mu(t)}{\lambda(t)}\right) \phi^{\prime}\left(\frac{x+\mu(t)}{\lambda(t)}\right) u^{2}(t, x) d x\right) d t<+\infty .
$$

This proves (1.40). 
Remark 5.1 (Final remark). Estimate (1.40) also reveals that the choice of $\tilde{\phi}$ in (5.4) is in some sense necessary for having truly decay. Indeed, from (5.6), having

$$
\frac{x+\mu(t)}{\lambda(t)} \gtrsim \frac{1}{\log t} \Longrightarrow x \gtrsim-t+t \log ^{\epsilon} t \sim t \log ^{\epsilon} t
$$

and now (5.6) becomes the more tractable integral estimate

$$
\int_{10}^{\infty} \frac{1}{t \log t}\left(\int_{t \log ^{\epsilon} t}^{t \log ^{1+\epsilon} t-t} \phi^{\prime}\left(\frac{x+\mu(t)}{\lambda(t)}\right) u^{2}(t, x) d x\right) d t<+\infty .
$$

Now this integral does not contain the bad term $\frac{x+\mu(t)}{\lambda(t)}$, but we have lost all the solitonic region in the estimate of integrability in time, a property that makes sense with Theorem 1.6. This remark is also valid for $\mathrm{KdV}, \mathrm{mKdV}, \mathrm{BBM}$ and $\mathrm{BO}$.

\section{REFERENCES}

[1] L. Abdelouhab, J.-L. Bona, M. Felland, and J.-C. Saut, Nonlocal Models for nonlinear, dispersive waves, Physica D 40 (1989) 360-392.

[2] J. P. Albert, Positivity properties and uniqueness of solitary wave solutions of the intermediate long wave equation, in Evolution equations (Baton Rouge, LA, (1992)), 11-20, Lecture Notes in Pure and Appl. Math., 168, Dekker, New York, 1995.

[3] J. P. Albert and J.F. Toland, On the exact solutions of the intermediate long-wave equation, Diff. Int. Eq. 7 (3-4) (1994), 601-612.

[4] T. B. Benjamin, Internal waves of permanent form in fluids of great depth, J. Fluid Mech. 29 (1967) $559-592$.

[5] J. L. Bona, D. Lannes and J.-C. Saut, Asymptotic models for internal waves, J. Math. Pures. Appl. 89 (2008) 538-566.

[6] H. H. Chen, and Y. C. Lee, Internal-Wave Solitons of Fluids with Finite Depth, Phys. Rev. Lett. 43 (1979) 264

[7] W. Craig, P. Guyenne, and H. Kalisch, Hamiltonian long-wave expansions for free surfaces and interfaces, Comm. Pure. Appl. Math. 58 (2005)1587-1641.

[8] P. Isaza, F. Linares, and G.Ponce, On the propagation of regularity and decay of solutions to the $k$-generalized Korteweg-de Vries equation, Comm. PDE, 40 (2015) 1336-1364.

[9] P. Isaza, F. Linares, and G. Ponce, On the propagation of regularities in solutions of the Benjamin-Ono equation, J. Funct. Anal. 270 (2016) 976-1000.

[10] R. I. Joseph, Solitary waves in a finite depth fluid, J. Phys. A 11 (1978) L97.

[11] R. I. Joseph,and R. Egri Multi-soliton solutions in a finite depth fluid, J. Phys. A 10 (1977) L225

[12] C. E. Kenig, and Y. Martel, Asymptotic stability of solitons for the Benjamin-Ono equation, Rev. Mat. Iberoam. 25 (2009) 909-970.

[13] C. E. Kenig, G. Ponce, and L. Vega, Well-posedness and scattering results for the generalized Kortewegde Vries equation via contraction principle, Comm. Pure Appl. Math. 46 (1993), 527-620.

[14] J. Klipfel, P. A. Perry and Yulin Wu, Work in progress.

[15] Y. Kodama, M. J. Ablowitz, and J. Satsuma, Direct and inverse inverse scattering problems of the nonlinear intermediate long wave equations J. Math. Phys. 23 (1982) 564-576.

[16] Y. Kodama, J. Satsuma, and M. J. Ablowitz, Nonlinear intermediate long-wave equation: analysis and method of solution, Phys. Rev. lett. (40) (1981), 687-690. 
[17] D. J. Korteweg and G. de Vries On the change of form of long waves advancing in a rectangular canal, and on a new type of long stationary waves, Philos. Mag. 39 (1895), 422-443.

[18] M. Kowalczyk, Y. Martel, and C. Muñoz, Kink dynamics in the $\phi^{4}$ model: asymptotic stability for odd perturbations in the energy space, J. Amer. Math. Soc. 30 (2017), 769-798.

[19] M. Kowalczyk, Y. Martel, and C. Muñoz, Nonexistence of small, odd breathers for a class of nonlinear wave equations, Letters in Mathematical Physics, (2017) Vol. 107, Issue 5, 921-931.

[20] C. Kwak, and C. Muñoz, Extended decay properties for generalized BBM equations, to appear in "Nonlinear Dispersive Partial Equations and Inverse Scattering", Peter D. Miller, Peter Perry, JeanClaude Saut, Catherine Sulem Eds, Fields Institute Communications 83, Springer (2019).

[21] T. Kubota, D. R. S. Ko, and L. D. Dobbs, Weakly nonlinear, long internal gravity waves in stratified fluids of finite depth, J. Hydronautics 12 (1978) 157-165.

[22] C. Kwak, C. Muñoz, F. Poblete, and J. C. Pozo, The scattering problem for the Hamiltonian abcd Boussinesq system in the energy space, J. Math. Pures Appl. (9) 127 (2019), 121-159.

[23] D. R Lebedev and A. O. Radul, Generalized internal long waves equations, construction, Hamiltonian structure, and conservation laws, Commun. Math. Phys. 91 (1983) 543-555.

[24] Y. Matsuno, Bilinear Transformation Method, Academic Press, New York, 1984.

[25] L. Molinet, and S. Vento, Improvement of the energy method for strongly nonresonant dispersive equations and applications, Anal. PDE 8 (2015), no. 6, 1455-1495

[26] C. Muñoz and G. Ponce, Breathers and dynamics of solutions in KdV type equations, Comm. Math. Phys. 367 (2019), 581-598.

[27] C. Muñoz and G. Ponce, On the asymptotic behavior of solutions to the Benjamin-Ono equation, to appear in Proc. Amer. Math. Soc. arXiv:1810.02329.

[28] H. Ono, Algebraic solitary waves on stratified fluids, J. Phy. Soc. Japan 39 (1975) 1082-1091.

[29] P. Santini, M. J. Ablowitz, and A.S. . Fokas, On the limit from the intermediate long wave equation to the Benjamin-Ono equation, J. Math. Phys. 25 (4) (1984), 892-899.

[30] J. Satsuma, M. J. Ablowitz, and Y. Kodama, On an internal wave equation describing a stratified fluid with finite depth Phys. Lett. A, 73 (1979) 283.

[31] J.-C. Saut, Benjamin-Ono and intermediate long wave equations : modeling, IST and PDE, arXiv : 1811.08652v1 21 Nov 2018, to appear in "Nonlinear Dispersive Partial Equations and Inverse Scattering", Peter D. Miller, Peter Perry, Jean-Claude Saut, Catherine Sulem Eds, Fields Institute Communications 83, Springer (2019).

[32] Li Xu, Intermediate long waves systems for internal waves, Nonlinearity 25 (2012), 597-640.

CNRS and Departamento de Ingeniería Matemática Dim-CMM UMi 2807-CNRS, UniverSidad de Chile, Santiago, Chile

E-mail address: cmunoz@dim.uchile.cl

Laboratoire de Mathmatiques, UMR 8628 Universit Paris-Sud et CNRS, 91405 Orsay, FRANCE

E-mail address: jean-claude.saut@u-psud.fr

Department of Mathematics, University of California, Santa Barbara, CA 93106. USA

E-mail address: ponce@math.ucsb.edu 